\title{
Impedance Microbiology-on-a-Chip: Microfluidic Bioprocessor for Rapid Detection of Bacterial Metabolism
}

\author{
Rafael Gómez-Sjöberg, Member, IEEE, Dallas T. Morisette, and Rashid Bashir, Senior Member, IEEE
}

\begin{abstract}
Detection of a few live bacterial cells in many industrial or clinical samples is a very important technological problem. We have developed a microscale technique for concentrating bacterial cells from a dilute sample, by factors on the order of $10^{4}$ to $10^{5}$, and detecting their metabolic activity by purely electrical means. The technique was implemented on a silicon-based microfluidic chip where the cells are concentrated and incubated in a chamber with a volume of $400 \mathrm{pL}$. Concentration and capture are obtained by the use of dielectrophoresis on the bacterial cells, and metabolism detection is achieved by means of impedance measurements of the medium in which the bacteria are incubated. Performing impedance-based detection at the microscale results in drastically reduced detection times for dilute bacterial samples, thanks to the ability to efficiently concentrate and capture the cells in an extremely small volume. Such concentration eliminates the need to amplify the bacterial population by long culture steps. This detection technique can be used for a wide variety of applications.
\end{abstract}

[1386]

Index Terms-Bacterial detection, bacterial metabolism, biochip, impedance microbiology.

\section{INTRODUCTION}

D ETECTION of a few live bacterial cells in clinical samples, water, pharmaceuticals, food, or cosmetics (and the raw materials used to produce these) is a very important technological problem. Food, cosmetic, and drug manufacturers must screen their raw materials and/or finished products for total bacterial content before being used for manufacturing, or shipped to customers, respectively. In the industries mentioned above, materials that are likely to contain pathogens or other undesired microorganizms are often subjected to various sterilization procedures. In these cases the purpose of screening is to ensure that no live bacteria remain after sterilization, so the detection method should only be sensitive to live cells. Currently, the official food screening procedures established by regulatory agen-

Manuscript received July 23, 2004; revised November 10, 2004. This work was supported by a cooperative agreement with the Agricultural Research Service of the U.S. Department of Agriculture, Project Number 1935-42000-03500D, through the Center for Food Safety Engineering at Purdue University. Competing Interests Statement: R. Bashir and D. T. Morisette declare competing financial interests. Subject Editor A. J. Ricco.

R. Gómez-Sjöberg was with the School of Electrical and Computer Engineering, Purdue University, West Lafayette, IN 47907 USA. He is now with the Department of Bioengineering, Stanford University, Stanford, CA 94305 USA.

D. T. Morisette is with BioVitesse, Inc., San Jose, CA 95129 USA.

R. Bashir is with Birck Nanotechnology Center and Bindley Biosciences Center in Discovery Park and School of Electrical and Computer Engineering and Weldon School of Biomedical Engineering at Purdue University, West Lafayette, IN 47907 USA, and also affiliated with BioVitesse, Inc. (e-mail: bashir@ecn.purdue.edu).

Digital Object Identifier 10.1109/JMEMS.2005.845444 cies [Food and Drug Administration (FDA) and U.S. Department of Agriculture (USDA)] are very sensitive, but require a long time to complete (up to $7 \mathrm{~d}$ ) with the work of highly skilled laboratory personnel [1], [2]. By the time a pathogen is identified in a product or its raw materials, the product would probably have been sold and used. The same is true for raw materials used for production of pharmaceuticals, where the current standard for purified water for final products specifies a maximum bacterial content of $100 \mathrm{cfu} / \mathrm{mL}$ (colony-forming-units per $\mathrm{mL}$ ) [3]. The most common sample volume used by the pharmaceutical industry is $100 \mathrm{~mL}$, which means that the screening protocol should be able to detect 10000 cfu total. This is, in fact, the target cell count that we have chosen for testing our detection technique. Few automated methods exist with sensitivity and reliability comparable to those of the manual methods; and the most sensitive automated techniques cannot discern between live and dead cells. To identify live bacteria with high sensitivity all current detection methods rely on a $24-48 \mathrm{~h}$ growth step to amplify the population of live cells.

One common automated bacterial detection method is based on the changes in the electrical characteristics of a medium where bacteria are cultured. These changes are produced by the release of ionic metabolites from live cells, measured by electrodes in contact with the medium [4]. The most common embodiment of this technique, commonly called "Impedance Microbiology," involves monitoring over time the ac impedance of a pair of electrodes immersed in the culture medium, at a single frequency. If the impedance changes beyond a certain threshold, a positive detection is indicated. This technique was first identified more than $100 \mathrm{yr}$ ago, and has been used for many years in bacterial growth monitoring, bacterial load control on perishable products, and bacterial detection. There are numerous reports in the literature dealing with its application to rapid bacterial detection [5]-[7]. And equipment for automated impedance monitoring ranging from laboratory, bench-top size up to industrial scale is commercially available from several vendors.

Unfortunately, the detection time of the conventional impedance-based method can be quite long when the concentration of bacterial cells present in the sample is very small [4]. The lower the initial concentration of microorganizms, the longer it takes for the impedance to change by a measurable amount. This limitation can be overcome if those few cells are confined into a very small volume while the impedance is being measured. In this way, the effective cell concentration is increased without increasing the number of cells. As we proposed in a previous publication [8], this concept leads 


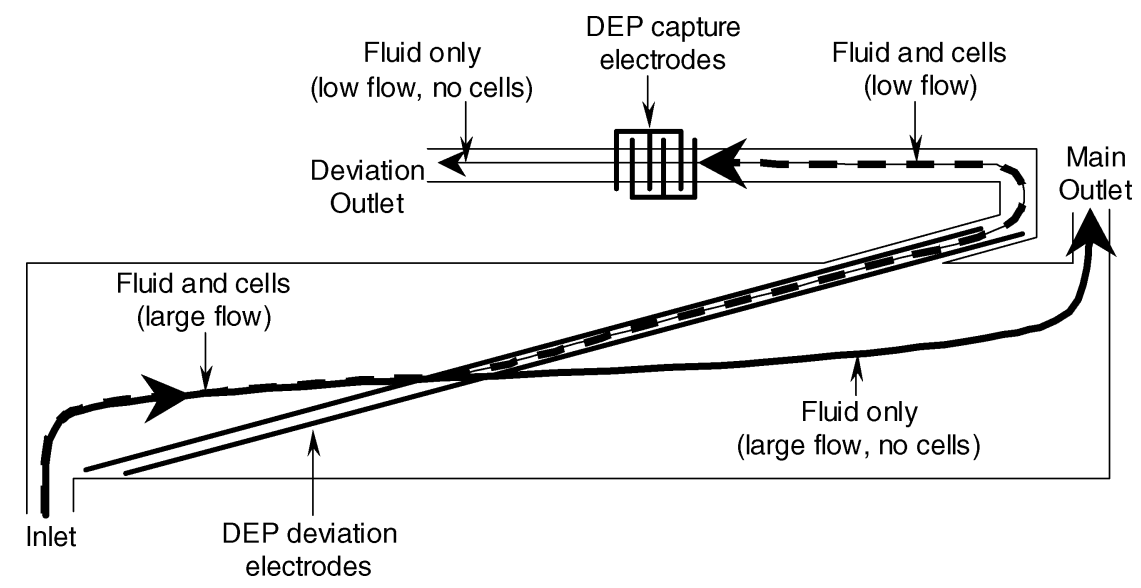

Fig. 1. Principle of operation of the dielectrophoresis-based deviation and capture of cells in the bioprocessor.

to the idea of implementing the impedance-based detection in a microfabricated device, where volumes on the order of nanoliters or picoliters can be easily created. Such application of microfabrication technologies seems especially fitting, since miniaturization of the impedance-based assay has the potential to directly reduce the time for detection by a significant factor. A microfabricated device capable of concentrating cells from a dilute sample completely eliminates the need for amplifying the bacterial population by growth. This new technique of "Impedance Microbiology-on-a-Chip" can be used not only in the front end of a system for determining the presence of live microorganizms in a sample, but for many applications in which a bacterial culture needs to be monitored.

In previous publications [8], [9], we described preliminary experiments designed to test the feasibility of the proposed detection method. In this paper, we present results of tests of the microscale impedance-based technique for real-time detection of bacterial metabolic activity and growth using two microfabricated devices with two types of bacteria: Listeria innocua, a nonpathogenic species of the genus Listeria, and Listeria monocytogenes, a pathogenic species of the same genus. L. innocua and $L$. monocytogenes are very closely related and are often found together in certain food products [10]. However, the technique presented here is not exclusive to Listeria cells and can be used with any other culturable microorganizm, including most yeasts and molds.

\section{MicRofluidic DeVICES}

\section{A. Device Design}

Two silicon-based microfluidic biochips were fabricated for testing the proposed detection scheme. The design and fabrication of the first biochip was described in detail somewhere else [9]. This first biochip has rectangular chambers connected in series by channels, both etched to a depth of $12 \mu \mathrm{m}$ onto a crystalline silicon substrate. The chambers have volumes between approximately $75 \mathrm{pL}$ and $5.3 \mathrm{~nL}$. Interdigitated platinum electrodes were created at the bottom of the chambers to measure the metabolism of cells injected into the chambers.

The second biochip or bioprocessor was designed to collect and concentrate the cells from a liquid sample, and detect their metabolism and growth as they are incubated inside the chip.
The overall design concept is based on a large straight channel (main channel) through which the sample can be flowed at the desired rate while the cells contained in it are deviated by dielectrophoretic (DEP) forces [11], [12] into a small channel that leads into the incubation/measurement chamber, which has a volume of $400 \mathrm{pl}$. In the incubation chamber the cells are retained and concentrated by DEP, and their metabolism is measured by platinum interdigitated electrodes in direct contact with the liquid in the chamber. In both the main channel and the incubation chamber, the DEP field is generated by interdigitated electrodes buried under a silicon dioxide layer and excited with separate ac voltage sources. A schematic diagram of the deviation and capture process is shown in Fig. 1. The main channel has a cross-sectional area large enough to accommodate a maximum flow rate of approximately $2 \mu \mathrm{L} / \mathrm{min}$ while keeping the peak fluid velocity low enough to guarantee that the DEP forces are adequate for deviating all of the cells into the incubation chamber. The DEP excitation voltage is limited to $20 \mathrm{Vpp}$ for two practical reasons. First, $20 \mathrm{Vpp}$ is the highest voltage that most bench-top signal generators can produce at the required frequencies. Second, at higher voltages the power dissipated into the chip by the DEP excitation signal is enough to raise its temperature beyond $100^{\circ} \mathrm{C}$. To ensure a very high deviation efficiency at a DEP excitation voltage of $20 \mathrm{Vpp}$ or less, the cells are deviated at a very shallow angle with respect to the long axis of the channel. The flow rate in the deviation channel is externally controlled between 0 and approximately $10 \mathrm{~nL} / \mathrm{min}$, independently of the flow rate in the main channel. To accurately measure the temperature in the chip, a platinum resistive-temperature-detector (RTD) is built into it, made of a meandering platinum resistor. Each biochip contains two identical concentration and detection devices, so that two tests can be performed in parallel, or one of the devices can be used for control or reference measurements while the other receives the sample to be tested.

\section{B. Bioprocessor Fabrication and Packaging}

The fabrication process of the bioprocessor started with bare 4" silicon wafers, with a (100) surface and a thickness of 500 $\mu \mathrm{m}$. Silicon dioxide was thermally grown on the wafers and subsequently patterned with conventional photolithography 


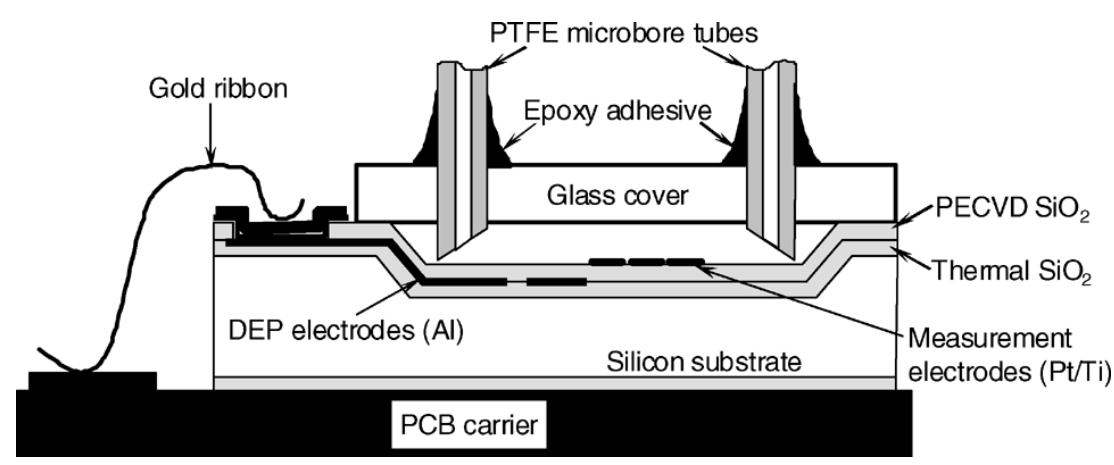

Fig. 2. Simplified cross section of a packaged bioprocessor.

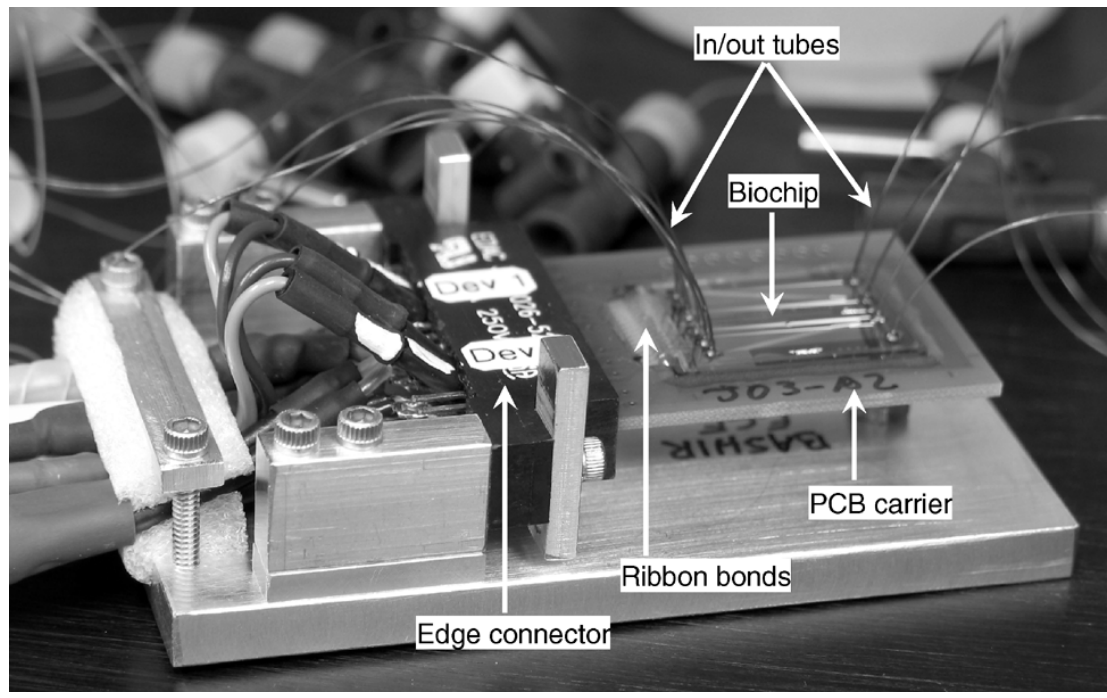

Fig. 3. Packaged bioprocessor connected to the measurement and control system.

(using Clariant AZ1518 positive photoresist, Clariant Corp., Somerville, NJ) followed by etching in buffered hydrofluoric acid (BHF). This oxide layer serves as a hard mask for etching the channels in an anisotropic potassium-hydroxide-based etchant to a nominal depth of $12 \mu \mathrm{m}$. After etching the channels, the hard mask was removed by etching in BHF and the wafers were thermally reoxidized to create a $2000-\AA$ layer of silicon dioxide. Over this oxide, a first metal layer, for the DEP electrodes, was deposited by sputtering of aluminum to a thickness of $1000 \AA$ (MRC-903 Sputterer, Materials Research Corporation, Orangeburg, NY) and patterned by liftoff to create the DEP electrodes (conventional lithography using Clariant AZ4620 positive photoresist, which was used for the remainder of the fabrication process). After patterning the aluminum, a 3500-Å-thick layer of silicon dioxide was deposited by plasma enhanced chemical vapor deposition (PECVD, STS-310PC, Surface Technology Systems plc, Newport, U.K.) to completely isolate the DEP electrodes and prevent electrolysis of the liquid in the channels. Windows were defined in the PECVD oxide layer using conventional lithography and opened by reactive-ion-etching (STS-340, Surface Technology Systems plc) to access the bond pads on the first metal layer. Subsequently, the second metal layer, which creates the measurement electrodes and the RTD temperature sensor, was deposited by sputtering
$800 \AA$ of platinum over a titanium adhesion layer (Perkin-Elmer Sputterer Model 2400, Perkin-Elmer Inc., Wellesley, MA) and patterned by liftoff. Subsequently, a $10000-\AA$-thick layer of gold over a titanium adhesion layer were deposited by electron-beam evaporation (Varian Inc., Palo Alto, CA) and patterned by wet etch to create robust bond-pads. After dicing the wafers, a glass cover was anodically bonded to each die at $400{ }^{\circ} \mathrm{C}$ with a voltage of $1000 \mathrm{~V}$ for $60 \mathrm{~min}$. The glass cover was made from 4", 500- $\mu \mathrm{m}$-thick polished Pyrex glass wafers type 7740 (Corning Inc., Corning, NY), which were custom diced and ultrasonically drilled to create holes where input/output tubes were attached. The holes in the glass were aligned to the input/output channels in the die before anodic bonding. Fig. 2 shows a cross section of the packaged device.

After fabrication, each die was fixed onto a custom-designed printed circuit board carrier that allows it to be easily connected to the equipment that measures the impedance, generates the DEP signals, and measures and controls the temperature. The carrier contains an integrated heater and gold-plated bond pads that connect to the pads on the chip by wire bonding. Teflon microbore tubes were inserted into the holes in the glass cover and permanently attached using epoxy adhesive. Fig. 3 shows a packaged bioprocessor mounted on a microscope stage and connected to the measurement and control system. 


\section{EXPERIMENTAL SETUP}

All fluids were injected into the chips using pressurized nitrogen at pressures between 0 and $552 \mathrm{kPa}(80 \mathrm{psi})$. The flow rate in the main channel of the bioprocessor was controlled by adjusting the nitrogen pressure applied at the injector. The flow rate through the incubation chamber was controlled by applying a back-pressure at the chamber output using pressurized nitrogen. In the first biochip, external valves at the input and output tubes were used to isolate the chip from the injection system during incubation; while in the bioprocessor all the microbore tubes leading into and out of the chip were manually pinched to achieve isolation.

Experiments with the first biochip were performed with up to two chips mounted onto a custom-made heated platform with a built-in RTD temperature sensor. Electrical connection to the chips was established by using probers mounted on micromanipulators attached to the heated platform. The chip was observed with a low power stereo microscope. Experiments with the bioprocessor were done with the chip carrier mounted on the stage of a Nikon Eclipse E600 epi-fluorescence microscope (Nikon USA Corp., Melville, NY). During all the experiments, a computer controlled the temperature of the chips to within $\pm 0.2{ }^{\circ} \mathrm{C}$ or better using readings from the off- or on-chip RTD sensors, depending on the chip being used, to adjust the power delivered to the heaters on which the chips were mounted. The chip stages were completely enclosed to keep air drafts from disturbing the temperature.

The impedance of the electrodes in the chip was measured with an Agilent 4284A LCR meter (Agilent Technologies Inc., Palo Alto, CA) connected to the chip through an Agilent 34970A switching unit fitted with two Agilent 34905A RF multiplexer cards. The multiplexer cards allowed the sequential measurement of up to four sets of electrodes with the same LCR meter. All of these instruments were connected to a computer through a GPIB interface. The impedance measurement process and the chip temperature control were automated by custom LabView (National Instruments Corp., Austin, TX) virtual instruments. With this setup, it was possible to acquire impedance versus frequency curves (the complex impedance could be measured at multiple frequencies between $100 \mathrm{~Hz}$ and $1 \mathrm{MHz}$ ) from up to four pairs of electrodes at fixed time intervals for an indefinite length of time. In the first biochip, the impedance of interdigitated electrodes in a 5.27-nL chamber was measured at 52 frequencies logarithmically spaced between $100 \mathrm{~Hz}$ and $1 \mathrm{MHz}$, with a 50-mVpp voltage excitation. In the bioprocessor the impedance of interdigitated electrodes in the incubation chamber was measured at 51 frequencies logarithmically spaced between $100 \mathrm{~Hz}$ and $100 \mathrm{kHz}$, with a $150-\mathrm{mVpp}$ voltage excitation. Sinusoidal and square-wave DEP signals were generated by Agilent $33120 \mathrm{~A}$ synthetized signal generators.

\section{MATERIALS AND Methods}

The following materials and methods were employed for all the tests performed with the first biochip and the bioprocessor.

\section{A. Bacterial Growth Media}

The composition of Luria-Bertani broth (LB), used for bacterial culture, was $10 \mathrm{~g} / \mathrm{L}$ tryptone, $5 \mathrm{~g} / \mathrm{L}$ yeast extract (both from Difco Laboratories, Detroit, MI), and $3.3 \mathrm{~g} / \mathrm{L}$ dextrose in de-ionized water. The nominal conductivity of this medium was $2.2 \mathrm{mS} / \mathrm{cm}$. Half-LB (HLB) was prepared by mixing equal parts of LB and deionized water. The nominal conductivity of HLB was $1.1 \mathrm{mS} / \mathrm{cm}$. Listeria cells grow in LB to a maximum population on the order of $5 \times 10^{8} \mathrm{cfu} / \mathrm{mL}$ after incubation at $37^{\circ} \mathrm{C}$ for $16 \mathrm{~h}$. Listeria cells grow in HLB to a final population not more than $30 \%$ lower than that achieved in LB (data not shown).

\section{B. Preparation of Bacterial Suspensions}

L. monocytogenes $v 7$ and L. innocua F4248 cells were grown in $\mathrm{LB}$ at $37^{\circ} \mathrm{C}$ for at least $16 \mathrm{~h}$. After growth, the cells were washed by repeated centrifugation and resuspension in sterile HLB, LB, or DI water, depending on the experiment. Washing guaranteed that the cells were suspended in a medium completely free of any metabolic byproducts. If heat-killed cells were desired, an aliquot from the as-grown cells was placed in a water bath at $80^{\circ} \mathrm{C}$ for $20 \mathrm{~min}$ and then washed in the same way as live cells. When fluorescent cells were needed a $1 \mathrm{ml}$ aliquot from the as-grown live or heat-killed cells was mixed with about $1 \mu \mathrm{L}$ of the green fluorescent dye $\operatorname{DiOC}_{6}(3)$ (3,3'-dihexyloxacarboxyanine iodide, Molecular Probes Inc., Eugene, OR), diluted to $1 \mathrm{nM}$ in dimethyl sulphoxide (DMSO). The mixture was incubated at room temperature for $30 \mathrm{~min}$ to allow the cells to absorb the dye, and washed in the same way described above. The washed cells were diluted in sterile HLB, LB, or DI water, depending on the experiment, to the approximate concentration desired for injection into the chip. An aliquot from the diluted cells was diluted further and plated on Brain-Heart-Infusion (Difco Laboratories) agar plates. The plates were incubated at $37^{\circ} \mathrm{C}$ for $24 \mathrm{~h}$ and the resulting colonies were counted to determine the actual concentration of viable cells in the suspension injected into the chip.

\section{Immunomagnetic-Based Cell Capture}

L. monocytogenes $v 7$ cells were captured with antilisteria Dynabeads (Dynal Biotech, Norway). L. monocytogenes were cultured in LB broth at $30^{\circ} \mathrm{C}$ for $24 \mathrm{~h}$, achieving a concentration of approximately $5 \times 10^{8} \mathrm{cfu} / \mathrm{mL}$. Heat-killed cells were obtained by placing an aliquot from the as-grown culture in a water bath at $80^{\circ} \mathrm{C}$ for $20 \mathrm{~min}$. A $1-\mathrm{mL}$ aliquot from the culture (live or heat-killed) was centrifuged and resuspended in $1 \mathrm{ml}$ phosphate-buffer-saline (PBS), then mixed with $40 \mu \mathrm{L}$ of the stock bead suspension and incubated at room temperature for $10 \mathrm{~min}$. The beads were collected with a magnet, most of the liquid was removed, and the beads were resuspended in $1 \mathrm{~mL}$ PBS-Tween (PBS with $0.05 \% \mathrm{v} / \mathrm{v}$ Tween-20) and incubated at room temperature for $10 \mathrm{~min}$. This was repeated twice resuspending in $1 \mathrm{~mL}$ PBS-Tween, and twice again resuspending in $1 \mathrm{~mL} \mathrm{LB}$ broth.

\section{On-Chip Cell Concentration and Metabolism Detection}

All the experiments were performed with the bioprocessor heated to $(37 \pm 0.1){ }^{\circ} \mathrm{C}$. The cells suspended in deionized 


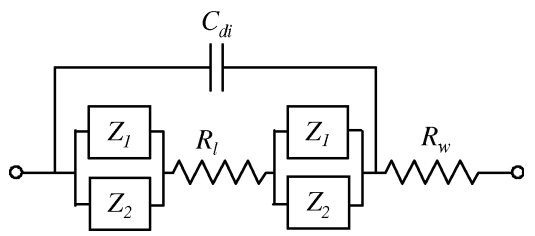

Fig. 4. Circuit model used to fit the impedance versus frequency curves obtained from the biochip.

water were injected at an input flow rate of approximately $1.7 \mu \mathrm{L} / \mathrm{min}$, with the deviation and capture electrodes excited with a $16 \mathrm{Vpp}$ square signal at $100 \mathrm{kHz}$. The broad spectrum of a square signal produced slightly higher DEP forces than a pure sinusoid at the same fundamental frequency. During injection, the flow rate in the incubation chamber was manually controlled to be between 4 and $10 \mathrm{~nL} / \mathrm{min}$. Flow rate control was performed manually by adjusting the pressures applied to the bioprocessor based on the observed velocity of the cells in the channels. After the whole $40 \mu \mathrm{L}$ of sample had been flowed through the bioprocessor the flow was stopped, the DEP deviation electrodes were turned off, and HLB was injected at a flow rate of less than $0.5 \mu \mathrm{L} / \mathrm{min}$. As the water in the chamber was replaced by HLB, the excitation voltage on the capture electrodes was increased to $20 \mathrm{Vpp}$ and the frequency to $3 \mathrm{MHz}$ to maximize the DEP forces acting on the cells. Once the incubation chamber was filled with HLB, the flow was stopped, all the microbore tubes leading into and out of the bioprocessor were pinched to seal them completely, and the capture electrodes were turned off. After pinching the tubes, the impedance measurement process was started and the cells were incubated for a minimum of $12 \mathrm{~h}$. When a reference measurement was desired, cells were injected only into one of the two devices in a bioprocessor, while the other device received sterile HLB only. The device with sterile media provided a baseline impedance in the absence of any metabolic activity.

\section{RESULTS}

\section{A. Preliminary Tests of On-Chip Bacterial Growth Detection}

The first biochip was used to perform preliminary tests of the impedance technique with live and heat-killed Listeria cells incubated inside the chip, suspended in Luria-Bertani broth (LB). Sterile LB and suspensions of different concentrations of $L$. innocua $F 4248$ cells were injected into the chip and incubated there at $(39 \pm 0.2){ }^{\circ} \mathrm{C}$ (temperature of the heating platform where the chip was mounted, which was estimated to result in an on-chip temperature of $\sim 37^{\circ} \mathrm{C}$ ) for more than $12 \mathrm{~h}$. The cells were uniformly distributed throughout the whole microfluidic path in the chip. The circuit model shown in Fig. 4 was fitted to each one of the measured impedance versus frequency curves (one curve per time point) to obtain a value for each of the model parameters at each time point. In the model, $C_{d i}$ accounts for the dielectric properties of all the materials surrounding the electrodes, $R_{l}$ represents the bulk resistance of the liquid, $R_{w}$ is the resistance of the on-chip wiring, and $Z_{1}$ and $Z_{2}$ are interfacial impedances given by the following expression [8], [13], [14]

$$
Z_{i}=\frac{1}{(j \omega)^{n_{i}} B_{i}}
$$
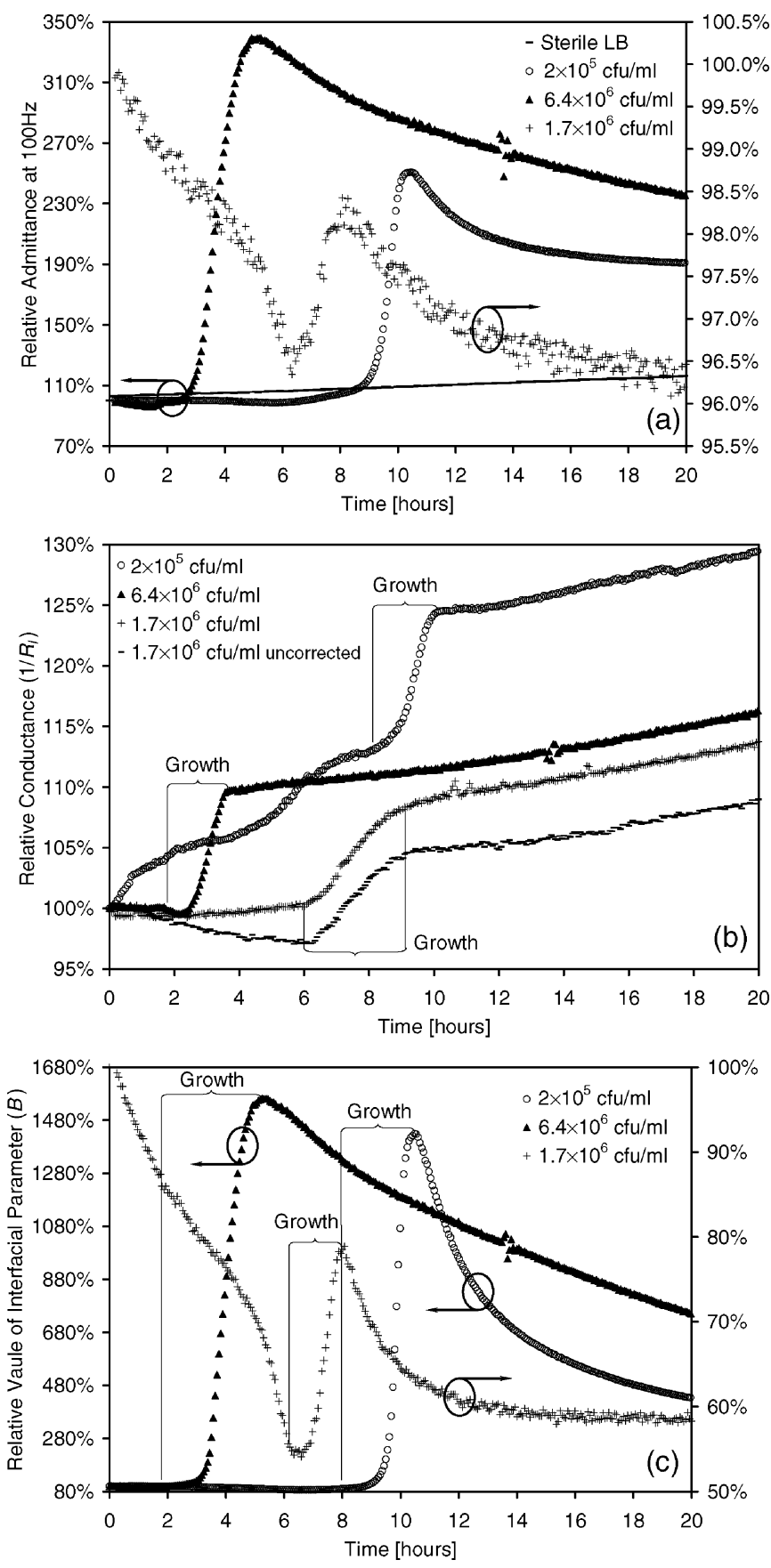

Fig. 5. (a) Relative admittance (reciprocal of the measured impedance) at $100 \mathrm{~Hz}$ of $L$. innocua suspensions at three different concentrations. (b) Relative conductance $G_{l}=1 / R_{l}$; temperature-corrected to $39^{\circ} \mathrm{C}$ (unless marked as "uncorrected" on the legend). (c) Relative value of the interfacial parameter $B$ [see (1)]. The sharp initial decrease in the curves corresponding to $1.7 \times 10^{6} \mathrm{cfu} / \mathrm{ml}$ was due a sharp decrease in temperature during the initial part of the incubation. Values at $t=0$ are defined as $100 \%$.

where $j=\sqrt{-1}$. This is the simplest model that would properly fit the measured data over the whole frequency range and at all times during incubation. It is speculated that the two different interfacial impedances account for the interactions of the electrodes with two distinct groups of species in the solution.

Fig. 5 shows the change over time in the admittance (reciprocal of the measured impedance) at $100 \mathrm{~Hz}$ [Fig. 5(a)], the conductance $G_{l}=1 / R_{l}$ [Fig. 5(b)], and the interfacial parameter $B_{i}$ corresponding to the parameter $n_{i}$ with the smallest 
value [Fig. 5(c)], for three different concentrations of $L$. inпосиа: $2 \times 10^{5}, 1.7 \times 10^{6}$, and $6.4 \times 10^{6} \mathrm{cfu} / \mathrm{mL}$. These concentrations correspond to approximately 1,9 , and $34 \mathrm{cfu}$, respectively, in the 5.27-nL chamber where the impedance was measured. The sharp initial drop in admittance and $B$ parameter corresponding to $1.7 \times 10^{6} \mathrm{cfu} / \mathrm{mL}$ was due to an accidental change in the temperature of the chip, from $40{ }^{\circ} \mathrm{C}$ to $38^{\circ} \mathrm{C}$, during the first $10 \mathrm{~h}$ of incubation. The conductance of all the samples was corrected for changes in temperature, to estimate its value at $39^{\circ} \mathrm{C}$, using a constant temperature variation coefficient estimated from the small random variations in temperature observed on the same data set. The $1.7 \times 10^{6} \mathrm{cfu} / \mathrm{mL}$ data with the accidental temperature change are included to illustrate the fact that, in spite of the sensitivity of the measurement to variations in temperature, it is possible to correct for those variations, at least in the conductance. It was not possible to find a simple temperature variation coefficient to correct the values of the interfacial parameter $B$, but we think that with further study of the issue it should be possible to find a suitable correction function (which might be nonlinear). With the exception of this single accidental temperature shift, the temperature control system was able to maintain the chip temperature within $\pm 0.2{ }^{\circ} \mathrm{C}$ of the set-point for more than $24 \mathrm{~h}$ in all other experiments.

With the exception of the temperature-induced drop in the data from one sample, the shape of the admittance and conductance versus time curves matches very well the typical shape of a bacterial growth curve. There is an initial lag phase where the cells are metabolizing, often at a slow rate, but not multiplying; during this period the impedance changes very little because the bacterial population is small. The lag phase is followed by a growth phase where the cells multiply exponentially. Such fastgrowing cell population releases large amounts of metabolic byproducts that in turn produce large changes in impedance. When the nutrients in the medium are depleted and/or the concentration of metabolic byproducts reaches a certain threshold, the cells stop multiplying and their population remains relatively constant or starts to decrease very slowly as cells die of starvation or due to the toxicity of the accumulated metabolic byproducts. During this final phase the conductance changes linearly due to the linear accumulation of metabolic byproducts released by a constant cell population.

The bimodal increase in conductance observed in the $2 \times 10^{5} \mathrm{cfu} / \mathrm{mL}$ sample during the first $8 \mathrm{~h}$ of incubation cannot be easily explained except probably by ionic contamination coming into the chamber. But this unexplained change serves to illustrate a very interesting phenomenon. During the exponential growth phase, the $B$ parameter corresponding to the $n$ parameter with the smallest value displays a very large increase (by up to factor of ten in some cases), followed by a slow decay. The other $B$ parameter does not change significantly during the course of the incubation. In the case of the $2 \times 10^{5} \mathrm{cfu} / \mathrm{mL}$ conductance curve, the changes observed during the first $8 \mathrm{~h}$ of incubation are very unlikely caused by bacterial growth because the relevant $B$ parameter does not change appreciably during this time. For detection purposes, monitoring the changes in the interfacial $B$ parameters, along with the changes in conductance, can improve the robustness of the method against changes in conductance not related to bacterial growth. It is suspected that some species released by the cells during growth rapidly adsorbs onto the surface of the electrodes and thus changes the interfacial impedance significantly. Such species would slowly degrade and/or desorb from the electrodes causing the slow decay in the $B$ parameter after exponential growth stops.

The use of interfacial parameters for impedance-based detection of bacterial growth had previously been discussed in [6], [7], and [15], but always fitting very simple circuit models, composed of standard resistors and capacitors, to the impedance measured at a single frequency. Such approach makes the circuit model completely disconnected from the actual phenomena taking place, especially because an infinite number of circuit models would match the measured impedance at a single frequency. The model presented here is derived from a physical analysis of the simplest electrochemical processes taking place at the interface and is, thus, bound to better represent the phenomena being measured. In addition, fitting the model to impedance values at a large number of frequencies over a large range, makes the measurement more sensitive. Such fitting essentially "concentrates" small changes in the impedance at each one of the frequency points into larger changes in a small number of parameters that might have some physical relevance.

Live and heat-killed L. monocytogenes $v 7$ cells from pure suspensions were used to test the metabolism detection process on bacteria captured by magnetic immunobeads. The captured cells plus beads were incubated on-chip in LB broth (biochip heater set to $39^{\circ} \mathrm{C}$ ). The impedance was measured during incubation in the same way as in the previous experiments. The concentration of beads injected into the chip was $1.7 \times 10^{7} \mathrm{~mL}^{-1}$. Preliminary tests of capture following the same procedure indicate that most of the beads carry at least one cell (data not shown), but the exact number of cells per bead is not known. Approximately 81 beads with live cells and 87 beads with heat-killed cells were manually counted in the 5.27-nL chamber where impedance was measured during the two experiments performed. Fig. 6 shows the admittance curves resulting from these cell incubations plus the incubation of sterile LB. The curve for live cells shows very clearly the three phases of cell growth, while dead cells and sterile media produce very small changes in admittance, as was expected from the absence of metabolism in the latter two samples.

\section{B. On-Chip Bacterial Concentration and Growth Detection}

To achieve a highly efficient concentration and capture process, the DEP forces in the deviation and capture stages of the bioprocessor have to be strong enough to counteract the drag forces exerted on the cells by the fluid around them. Testing of the concentration process in the bioprocessor was carried out with fluorescently labeled $L$. monocytogenes cells suspended in LB, HLB, and deionized water. The DEP forces on Listeria cells suspended in HLB or LB are too weak to achieve an efficient concentration process at reasonable flow rates. With the cells suspended in LB or HLB, the injection time would be on the order of tens of hours. However, Listeria cells experience very strong DEP forces when suspended in deionized water with a conductivity of $1 \mu \mathrm{S} / \mathrm{cm}$, at an excitation 


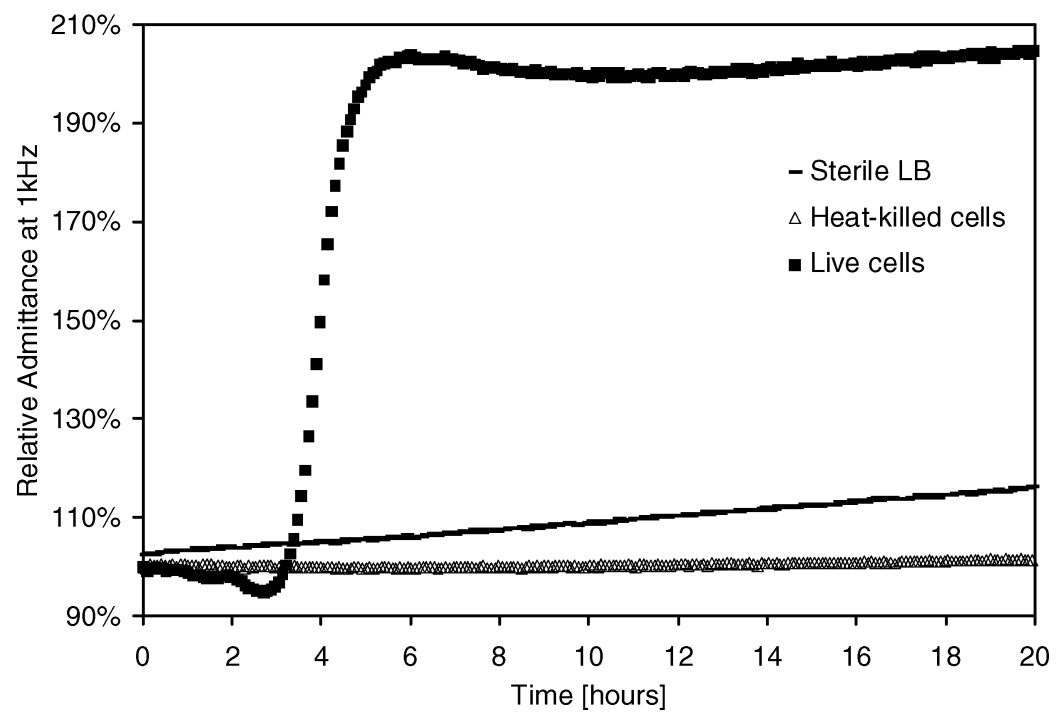

Fig. 6. Relative admittance of live and heat-killed L. monocytogenes cells captured by immunomagnetic beads. Values at $t=0$ are defined as $100 \%$.

frequency of $100 \mathrm{kHz}$. Under these conditions, the DEP forces are directed toward the regions where the electric field gradient is largest (positive DEP), so that the cells collect on the edges of the electrodes. Fig. 7(a) shows L. monocytogenes cells being deviated from the main channel toward the deviation channel. Fig. 7(b) shows cells concentrated in the incubation chamber in large accumulations over the gaps between the capture electrodes. With the right combination of medium conductivity, flow velocities, and DEP excitation voltage and frequency, the concentration efficiency is virtually $100 \%$.

To test the complete process of cell concentration and metabolism detection, fluorescently labeled L. monocytogenes $\checkmark 7$ cells, suspended in $40 \mu \mathrm{L}$ of sterile deionized water at concentrations of $(2.3 \pm 0.2) \times 10^{5},(6.8 \pm 0.4) \times 10^{5}$, and $(8.7 \pm 0.8) \times 10^{4} \mathrm{cfu} / \mathrm{mL}$, were injected into the chip at a flow rate of approximately $1.7 \mu \mathrm{L} / \mathrm{min}$ and incubated for at least $12 \mathrm{~h}$ at $37^{\circ} \mathrm{C}$. The total number of cells injected was approximately 9200,27200 , and 3480, respectively. During injection of the sample with $(2.3 \pm 0.2) \times 10^{5} \mathrm{cfu} / \mathrm{mL}$, the DEP electrodes were off so that the cells were not concentrated in the incubation chamber, resulting in a probability of approximately 0.09 of finding one cell in the chamber. The other two samples were injected with the DEP electrodes activated, which caused most of the cells in the samples to be collected in the incubation chamber. The actual number of cells collected is not known, but it was visually confirmed that only a very small fraction of the cells escaped the deviation and concentration process (no more than $\sim 10 \%$ ). During the switch from water to HLB a more significant fraction of the cells were lost because the DEP force was weakened by the increased medium conductivity coupled to instabilities in the flow rate, but thousands of cells could still be seen collected in the chamber, as shown in Fig. 7(b). Fig. 7(c) shows the change in admittance over time for the three L. monocytogenes samples plus sterile HLB. The frequencies chosen for plotting each curve, out of the 51 frequencies that were monitored, were those for which the relative impedance change was the largest. As expected, the sterile media did not exhibit any clear metabolic signal at any frequency. And the bacterial sample injected without the DEP-based concentration system active did not generate a clear metabolic signal corresponding to exponential growth for more than $7 \mathrm{~h}$ because the number of cells in the bioprocessor was very low. It is very likely that the linear increase in admittance observed in this sample, and the slow decrease seen in the sterile media, were due to a drift in the background admittance. Such drift was fairly common in any incubation of sterile media [see Fig. 5(a)]. After approximately $7.5 \mathrm{~h}$, the metabolic signal from the nonconcentrated sample is clear and its shape agrees well with the signal observed in previous incubations. On the other hand, in the two samples injected with the concentration system active a very strong metabolic signal was visible during the first hour of incubation.

\section{DISCUSSION}

The three experiments discussed above prove very clearly how the DEP-driven concentration system reduces dramatically the length of the detection process for very dilute bacterial suspensions. By collecting a large percentage of the cells from a very dilute sample in the 400-pL detection chamber, the deviation and capture system makes the effective cell concentration in the chamber orders of magnitude larger than that in the original sample. In the experiments presented, the concentration factor is between $10^{4}$ and $10^{5}$, which is the ratio of the original sample volume ( $40 \mu \mathrm{L})$ to the incubation chamber volume $(400 \mathrm{pL})$ times the fraction of cells that are permanently captured in the chamber, assuming that between $10 \%$ and $100 \%$ of the cells were captured. The exact concentration factor is difficult to estimate since it is impossible to count the cells remaining in the chamber, but it is almost certain that at least $10 \%$ of them remain. Most of the cell losses were caused by instabilities in the flow rate in the detection chamber, which could be eliminated with a better flow control system (using a syringe pump, for example). Even larger concentration factors can be achieved by injecting a larger sample, at the expense of a longer injection time. Increasing the concentration factor would allow the detection of bacteria in even more 

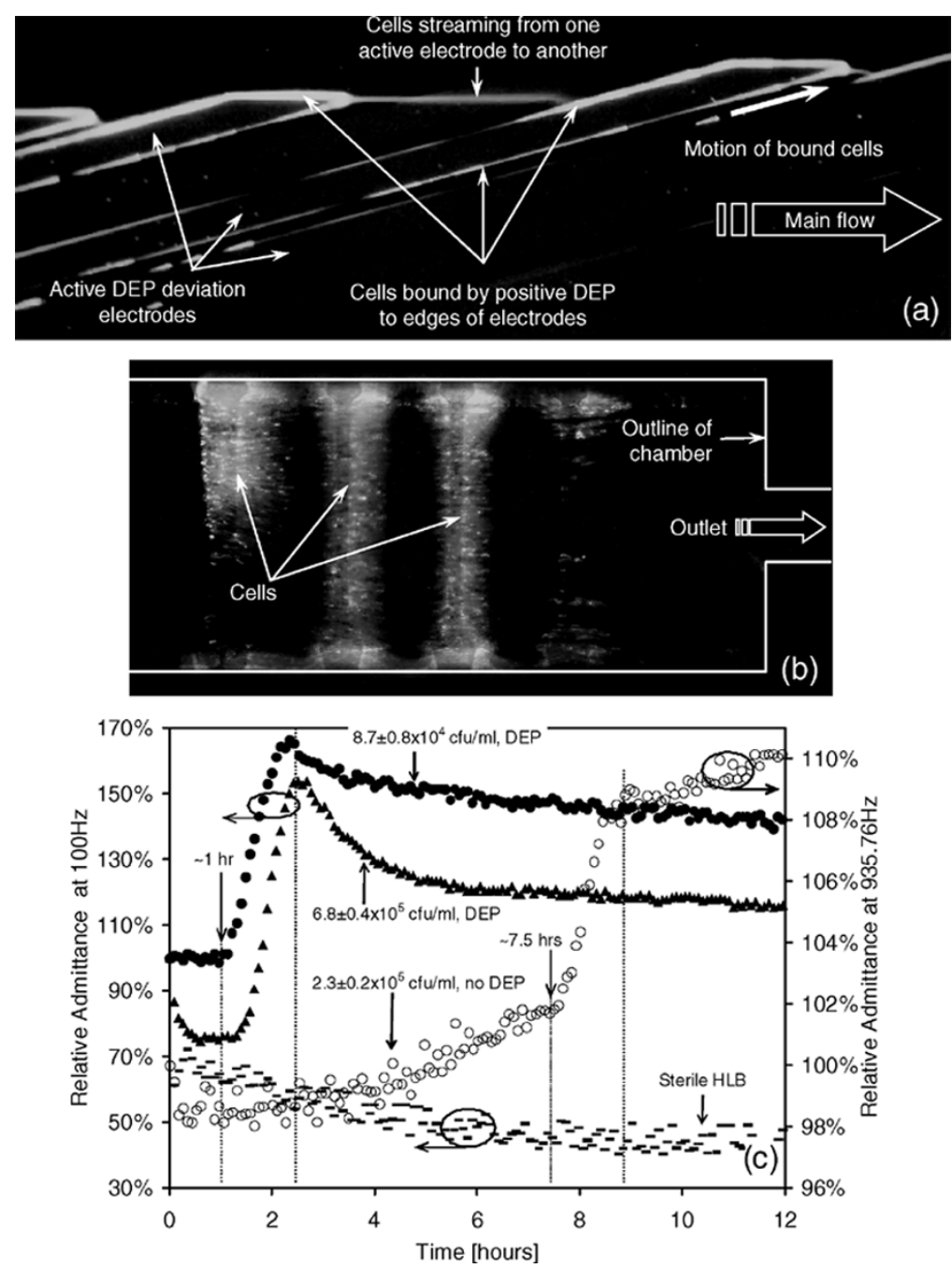

Fig. 7. (a) Fluorescently labeled L. monocytogenes cells being guided by the DEP electrodes out of the main channel and into the deviation channel that leads to the incubation chamber. All of the bright areas are accumulations of cells. (b) Cells concentrated from a suspension with $6.8 \times 10^{5} \mathrm{cfu} / \mathrm{mL}$ into the incubation chamber immediately before the start of incubation. The cells collect in the gaps between the capture electrodes. (c) Relative admittance of $L$. monocytogenes injected into the bioprocessor at various concentrations, with and without the DEP-based concentration system active, plus sterile HLB. Values at $t=0$ are defined as $100 \%$.

dilute samples. But even with the current concentration factor it would be possible to detect smaller numbers of bacteria than those tested. Unfortunately, it was not possible to use more dilute cell suspensions with the current setup because they made it impossible to control the flow rates through the device. Flow rate control was performed manually based on the observed velocity of the cells in the channels. Fewer cells in the channels would provide less feedback on the flow velocity. This problem can also be solved with a better flow control system that does not require manual adjustment.

The impedance measurement is fairly sensitive to temperature, as evidenced by the data shown, which proves the need for a robust and accurate temperature control system. With the exception of a single accidental temperature shift caused by a software malfunction, the temperature control system used in the first biochip was able to maintain the temperature within $\pm 0.2{ }^{\circ} \mathrm{C}$ of the set-point for more than $24 \mathrm{~h}$ in all other experiments. A more sophisticated temperature control system and better insulation can easily improve the temperature regulation to better than $\pm 0.1^{\circ} \mathrm{C}$. Temperature control is more accurate in the bioprocessor thanks to the on-chip RTD sensor, which is located only approximately $500 \mu \mathrm{m}$ from the measurement chambers so that it tracks the true chamber temperature much more closely. Additionally, the second detection chamber in the bioprocessor could also be used to correct for temperature-induced changes in impedance if it is filled with sterile incubation media as a negative control.

As was stated in the introduction, the target cell count that we have chosen for testing our detection technique is based on the specification for the total bacterial load in purified water for pharmaceutical production. The specification calls for less than $100 \mathrm{cfu} / \mathrm{mL}$ in a $100-\mathrm{mL}$ sample. Flowing such large sample volume through the bioprocessor would take a prohibitively large amount of time since the flow rate is limited to approximately $2 \mu \mathrm{L} / \mathrm{min}$ by the DEP concentration system. The solution for the handling of such large-volume samples involves the development of a filtration system that can concentrate the bacteria present in the samples and resuspend them in a $50-100 \mu \mathrm{L}$ volume [16] that can then be injected into the bioprocessor in a practical amount of time. 


\section{SUMMARY}

We have demonstrated a microfluidic technique for concentrating bacterial cells from a dilute sample, by factors on the order of $10^{4}$ to $10^{5}$, and detecting their metabolic activity by purely electrical means. Tests with dilute $L$. monocytogenes suspensions demonstrated the ability of this technique to significantly reduce the time needed to detect the presence of the bacteria. We have also explored the use of a physics-based circuit model fitted to impedance measurements at a large numbers of frequencies, as a means of improving the sensitivity and accuracy of the detection of metabolic activity. The measurement is very sensitive to temperature variations, which are minimized by an automatic temperature control system, but the data shown suggest that it is possible to correct for fairly large variations if they accidentally occur. The microscale application of impedance-based detection of bacteria coupled with a dielectrophoresis-based cell concentration system has the potential to dramatically decrease the time needed to screen industrial and clinical samples for total bacterial content, which can currently take several days using conventional methods.

\section{ACKNOWLEDGMENT}

The authors would like to thank Prof. M. R. Ladisch and Prof. A. K. Bhunia of Purdue University, and Dr. L. Razouk of BioVitesse, Inc., for useful discussions and helpful suggestions. BioVitesse, Inc., also acknowledges the support from the NSF through an SBIR Phase 1 and Phase 2 Award. They also acknowledge the assistance provided by K. Gray of the Department of Food Science, and the help received from D. Lubelski, T. Miller, B. Crabill, and C. Y. Fong of the microelectronics fabrication facilities at Purdue University, where most of the microfabrication process took place. Part of the fabrication process of the bioprocessor was carried out at the Microfabrication Applications Laboratory, University of Illinois, Chicago.

\section{REFERENCES}

[1] United States Food and Drug Administration, Bacteriological Analytical Manual, Rev. A, 8th ed. Gaithersburg, MD: Association of Official Analytical Chemists Int., 1998.

[2] "FSIS Method for the isolation and identification of listeria monocytogenes from processed meat and poultry products," U.S. Dept. Agriculture, Food Safety and Inspection Service, Laboratory Communication no. $57,1989$.

[3] The United States Pharmacopeia, USP1 \& USP2, XXII ed. Easton, PA: Mack, 1990.

[4] R. Eden and G. Eden, Impedance Microbiology. New York and U.K.: Research Studies-Wiley, 1984.

[5] M. Wawerla, A. Stolle, B. Schalch, and H. Eisgruber, "Impedance microbiology: Applications in food hygiene," J. Food Protection, vol. 62, no. 12 , pp. $1488-1496,1999$.

[6] L. Yang, C. Ruan, and Y. Li, "Detection of viable Salmonella typhimurium by impedance measurement of electrode capacitance and medium resistance," Biosens. Bioelectron., vol. 19, pp. 495-502, 2003.

[7] L. Yang, Y. Li, C. L. Griffis, and M. G. Johnson, "Interdigitated microelectrode (IME) impedance sensor for the detection of viable Salmonella typhimurium," Biosens. Bioelectron., vol. 19, pp. 1139-1147, 2004.

[8] R. Gómez, R. Bashir, and A. K. Bhunia, "Microscale electronic detection of bacterial metabolism," Sens. Actuators B-Chemical, vol. 86, no. 2-3, pp. 198-208, Sep. 2002.
[9] R. Gómez, R. Bashir, A. Sarikaya, M. R. Ladisch, J. Sturgis, J. P Robinson, T. Geng, A. K. Bhunia, H. L. Apple, and S. T. Wereley, "Microfluidic biochip for impedance spectroscopy of biological species," Biomed. Microdevices, vol. 3, no. 3, pp. 201-209, Sep. 2001.

[10] J. G. Amoril and A. K. Bhunia, "Immunological and cytopathogenic properties of Listeria monocytogenes isolated from naturally contaminated meats," J. Food Safety, vol. 19, pp. 195-207, 1999.

[11] H. A. Pohl, Dielectrophoresis - The Behavior of Neutral Matter in Nonuniform Electric Fields. Cambridge, New York, U.K.: Cambridge University Press, 1978.

[12] U. Seger, S. Gawad, R. Johann, A. Bertsch, and P. Renaud, "Cell immersion and cell dipping in microfluidic devices," Lab Chip, vol. 4, pp. 148-151, 2004.

[13] A. J. Bard and L. R. Faulkner, Electrochemical Methods. New York: Wiley, 1980.

[14] K. J. Vetter, Electrochemical Kinetics. New York: Academic, 1967.

[15] C. J. Felice, R. E. Madrid, J. M. Olivera, V. I. Rotger, and M. E. Valentinuzzi, "Impedance microbiology: Quantification of bacterial content in milk by means of capacitance growth curves," J. Microbiolog. Methods, vol. 35, pp. 37-42, 1999.

[16] W. Chen and M. R. Ladisch, private communication, Purdue University, Dep. Agricultural and Biological Eng., West Lafayette, IN, to be published.

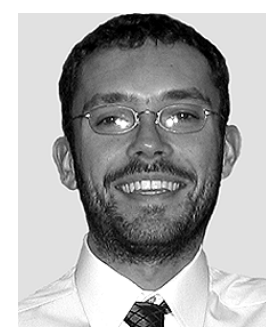

Rafael Gómez-Sjöberg (M'00) received the B.S. degree in electrical engineering from the University of Los Andes, Bogotá D.C., Colombia, in 1994, and the M.S.E.E. and Ph.D. degrees from the School of Electrical and Computer Engineering, Purdue University, West Lafayette, IN, in 1999 and 2003, respectively.

After receiving the B.S. degree, he was with the Ecole Nationale Supérieure des Télécommunications de Bretagne, Brest, France, designing and building optical and electronic instrumentation for research on liquid crystals. Subsequently, he worked on the D-Zero collaboration at the Fermi National Accelerator Laboratory, Batavia, IL, in the development of silicon- and scintillating optical fiber-based subatomic particle detectors, before enrolling in graduate school. During his graduate studies, he did a summer-long internship in the Advanced Technology Group at National Semiconductor Corporation, Santa Clara, CA, working on the development of submicron lateral bipolar transistors. After earning his doctorate, he worked as a Postdoctoral Research Scholar with the Department of Applied Physics, California Institute of Technology, Pasadena. He is currently a Postdoctoral Scholar with the Department of Bioengineering, Stanford University, CA. His current interests are biophysics, molecular biology, and the design and fabrication of microelectromechanical and microfluidic systems for biological and biomedical applications.

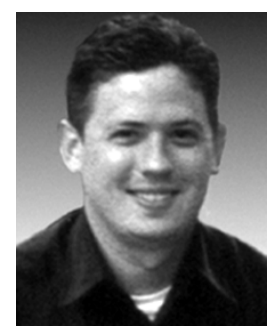

Dallas T. Morisette received the B.S. degree in electrical engineering from Walla Walla College, College Place, WA, in 1993, the M.S.E.E. degree from Purdue University, West Lafayette, IN, in 1997, and the Ph.D. degree also from Purdue University, for his work on the development of silicon carbide Schottky diodes in 2001 .

As an undergraduate, he was with G.E. Lighting Systems, Hendersonville, NC, where he developed a commercial lighting design and modeling software package. After receiving his doctorate, he was a $\mathrm{Di}$ rector of Product Development for OptoLynx, Inc., West Lafayette, IN, a developmental-stage telecommunications component manufacturer. In 2002, he returned to Purdue University as a principal research engineer, and is now a senior device engineer for BioVitesse, Inc., a biotechnology startup company based in West Lafayette, IN. 


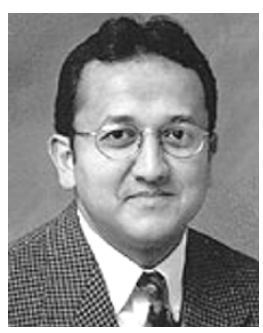

Rashid Bashir (SM'00) received the B.S.E.E. degree from Texas Tech University, Lubbock, as the highest ranking graduate in the College of Engineering in December 1987. He received the M.S.E.E. and Ph.D. degrees from Purdue University, West Lafayette, IN, in 1989 and 1992, respectively.

From October 1992 to October 1998, he was a Senior Engineering Manager working in the Process Technology Development Group, National Semiconductor Corp., Santa Clara, CA. His group worked on developing state-of-the-art bipolar and BiCMOS microelectronic fabrication processes for high voltage, analog and RF applications, SiGe HBT devices, SOI-bonded wafers, and MEMS technologies. He is currently an Associate Professor of Electrical and Computer Engineering at Purdue University since October 1998 and Associate Professor of Biomedical Engineering since June 2000. He has authored or coauthored more than 100 journal and conference papers and over 25 patents. His research interests include MEMS, BioMEMS, applications of semiconductor fabrication to biomedical engineering, advanced semiconductor fabrication techniques, and nanobiotechnology.

Dr. Bashir received the NSF Career Award for his work in Biosensors and BioMEMS in 2000. He also received the Joel and Spira Outstanding Teaching Award from the School of Electrical and Computer Engineering at Purdue University, and the Technology Translation Award from the 2001 BioMEMS and Nanobiotechnology World Congress Meeting, Columbus, OH. He was also selected by the National Academy of Engineering to attend the Frontiers in Engineering Workshop in fall 2003. 